\title{
Complicações Pós-Operatórias Cirúrgicas da Hemorroidectomia: Revisão de 76 Casos de Complicações
}

\author{
Surgical Complications of Hemorrhoidectomy: A Review of 76 Cases
}

\author{
GERALDO MAGELA GOMES DACRUZ ${ }^{1}$, SANDRA KELY ALVES DEALMEIDASANTANA ${ }^{1}$, \\ JORGE LUIZ SANTANA ${ }^{2}$, RENATAMAGALIRIBEIRO SILLUZIO FERREIRA ${ }^{3}$, PETERSON MARTINS NEVES $^{3}$, \\ MARINANEVES ZERBINIDEFARIA ${ }^{4}$
}

${ }^{1}$ Mestre, Doutor e Professor Titular de Coloproctologia; ${ }^{2}$ Pós-graduando lato sensu em Coloproctologia; ${ }^{3}$ Assistente do Grupo de Coloproctologia; ${ }^{4}$ Estagiária Voluntária do Serviço de Coloproctologia.

CRUZ GMG; SANTANA SKAA; SANTANA JL; FERREIRA RMRS; NEVES PM; FARIA MNZ. Complicações Pós-Operatórias Cirúrgicas da Hemorroidectomia: Revisão de 76 Casos de Complicações. Rev bras Coloproct, 2007;27(1): 042-057.

RESUMO: Dos 34.000 pacientes coloproctológicos foi feito o diagnóstico de doença hemorroidiana, como doença coloproctológica principal em 9.289 pacientes $(27,3 \%)$, dos quais $2.417(26,0 \%)$ foram submetidos à hemorroidectomia. A finalidade deste trabalho é analisar estes $\mathbf{7 6}$ casos de complicações à luz da idade e sexo dos pacientes e das técnicas cirúrgicas que a elas deram origens, bem como a abordagem de cada tipo de complicação, comparando os resultados com outros similares da literatura. A grande maioria dos pacientes $(2.014$ casos, $83,3 \%)$ foi submetida à técnica aberta, seguindo-se a técnica fechada (232 casos, $9,6 \%$ ) e a mista (171 casos, $7,1 \%)$, resultando 76 complicações $(3,1 \%)$, principalmente estenose anal cirúrgica $(44$ casos, $1,8 \%)$, hemorragia grave ( 21 casos, $0,9 \%$ ), seguindo-se agravamento da incontinência fecal e sepse anal grave (5 casos de cada, $0,2 \%)$ e complicações clínicas graves $(0,1 \%)$, sem óbitos. A análise dos dados da série de pacientes permitiu as seguintes conclusões. A estenose anal foi mais comum na quarta década (18 casos, 2,6\%), com hipertonia esfincteriana (70,0\%, 21 casos), de forma anular (70,4\%, 31 casos), entre 11 e 30 dias de pós-operatório (61,5\%, 27 casos), 31,8\% (14 casos) sendo abordada por anotomia simples e excisão de área de fibrose e anoplastia com retalho cutâneo em "V" (7 casos, 15,9\%). A hemorragia grave foi mais comum na terceira década (6 casos, 1,2\%), todos os pacientes foram submetidos à ligadura e transfixação de pedículo. Dos 5 casos de agravamento da incontinência fecal somente 2 pacientes $(40,0 \%)$ foram submetidos à esfincteroplastia "em jaquetão"; todos os 3 casos de sepse anal grave foram submetidos à drenagem e desbridamentos; e todas as 3 complicações clínicas graves foram abordadas clinicamente.

Descritores: Hemorróidas; hemorroidectomia; complicações da hemorroidectomia; complicações cirúrgicas anais.

\section{INTRODUÇÃO}

A doença hemorroidária $(\mathrm{DH})$ pode ser abordada por condutas clínicas ${ }^{37}$, intervencionistas não cirúrgicas $1,2,4,8,20,21,25,28,29,30,33,35,37,39,40,44,50,53$ e cirúrgicas $10,11,13,15,18,19,23,25,26,27,29,31,32,34,36,38,41,42,43,44,46$, $47,48,49,51,52$

O objetivo das várias modalidades de abordagens intervencionistas não-cirúrgicas é destruir os plexos hemorroidários, podendo-se citar entre eles: li- gadura elástica ${ }^{3,8,21,25,33,35,44,50,53}$, escleroterapia ${ }^{11,21,}$ 25,33 , crioterapia $11,12,21,25,28,30,33$, infrared ou fotocoagulação $11,25,30,33,40$, diatermia 1, 25, eletrocoagulação ${ }^{20,39}$.

O objetivo do tratamento cirúrgico na DH é a ressecção dos componentes internos e externos dos plexos hemorroidários e de tecidos envolvendo-os e comprometidos pelos mesmos. Apesar dos inúmeros epônimos cirúrgicos, há, na verdade, quatro técnicas básicas: técnicas abertas ${ }^{11,15,25,42}$, técnicas fechadas

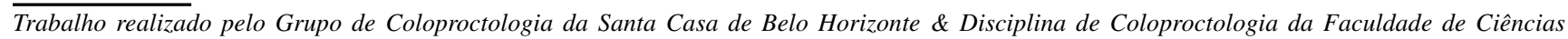
Médicas de Minas Gerais - Belo Horizonte - MG - Brasil. 
5, 11, 15, 25, 27, 42, técnicas mistas 5,34,36, 43 e técnicas amputativas (técnica de Whitehead e PPH) ${ }^{52}$. Todas estas cirurgias, tradicionalmente realizadas em regime hospitalar, hoje podem ser executadas também ambulatorialmente $11,18,22,23,38,41,44,48$. Da mesma forma, além do tradicional bisturi e da tradicional tesoura, podem, também, ser realizadas a laser (laser de $\mathrm{CO}_{2} \mathrm{e}$ o Nd:YAG laser) ${ }^{11,18,19,23,25,46,47,51 .}$.

Alguns nomes ficaram definitivamente e historicamente ligados às cirurgias para a DH, quer seja por terem criado técnicas como a aberta (MilliganMorgan ${ }^{29}$ ), a fechada (Ferguson ${ }^{13}$ ), e circunferencial ou amputativa (Whitehead ${ }^{52}$ ), quer seja por terem aperfeiçoado técnicas, como Parks ${ }^{36}$, Ruiz Moreno ${ }^{43}$, Sokol ${ }^{5}$ e Obando ${ }^{34}$.

Dentre as grandes séries registradas na literatura merecem destaques especiais às séries de Ganchrow et al. ${ }^{14}$ com 2.038 casos , a série de Iwagaki et al. ${ }^{19}$ com 1.816 casos e a série de Santos ${ }^{44,45}$ com 1.327 casos.

As complicações mais freqüentes da DH relatadas na literatura são: trombose, estrangulamento, hemorragia, ulceração com infecção secundária, e menos freqüentemente necrose, abscesso e incontinência fecal 10, 11, 13, 15, 18, 19, 23, 25, 26, 27, 29, 31, 32, 34, 36, 38, 41, 42, 43, 44, 46, 47, 48, 49, 51, 52. Santos 44,45 , em sua série de 1.327 pacientes abordados por DH chama a atenção para a confusão entre trombose hemorroidária em portadores de $\mathrm{DH}$ e simples hematomas anais e perianais de origem traumática, que soem acontecer mesmo em pacientes não portadores de DH.

Ganchrow et al. ${ }^{14,}$ em sua série de 2.038 pacientes operados agrupam as complicações pós-operatórias em imediatas (82 casos, 4,0\%) e tardias (99 casos, 4,8\%), contabilizando complicações sistêmicas, desde pneumonias até cistites. Atendo-se apenas às complicações anais foram as seguintes as incidências: fissura anal (54 casos, 2,7\%), estenose anal (24 casos, $1,2 \%)$, hemorragia cirúrgica 27 casos $(1,3 \%)$, abscesso anal (17 casos, 0,8\%), fístula anal (5 casos, $0,2 \%)$, totalizando 127 complicações anais $(6,2 \%)$.

\section{OBJETIVO}

O objetivo deste trabalho é estudar 76 casos de complicações pós-operatórias decorrentes de 2.417 pacientes submetidos à hemorroidectomia, analisando suas ocorrências em relação às técnicas cirúrgicas de hemorroidectomia usadas, idade e sexo dos pacientes, além de abordar as várias características e opções terapêuticas das várias complicações observadas estenose anal, hemorragia anal, agravamento da incontinência fecal, sepse anal e complicações sistêmicas (tabela A).

\section{CASUÍSTICA - PACIENTES E MÉTODO}

Dos 34.000 pacientes coloproctológicos foi feito o diagnóstico de DH como doença coloproctológica principal em 9.289 pacientes $(27,3 \%)$, dos quais 2.417 (26,0\%) foram submetidos à hemorroidectomia, decorrendo 76 complicações $(3,1 \%)$ (tabela A). É sobre estes 76 casos de complicações que se refere este trabalho.

\section{RESULTADOS}

Incidência absoluta de complicações póshemorroidectomia:

Dos 9.289 pacientes portadores de DH entre os 34.000 pacientes submetidos ao exame proctológico $(27,3 \%), 2.417(26,0 \%)$ foram submetidos à hemorroidectomia, decorrendo 76 complicações $(3,1 \%)$ (tabela A).

Incidência absoluta hemorroidectomia e de complicações pós-hemorroidectomia de acordo com as técnicas cirúrgicas empregadas (aberta, fechada e mista):

Dos 2.417 pacientes submetidos à hemorroidectomia resultaram 76 complicações $(3,1 \%)$ : 2.014 pacientes foram operados por técnica aberta ou Milligan-Morgan (83,3\%), levando a 61 casos de complicações $(3,0 \%) ; 232$ pacientes foram operados pela técnica fechada ou de Ferguson $(9,6 \%)$, levando a 8

Tabela A - Números absolutos (N) e percentuais (\%) de pacientes portadores de doença hemorroidária (DH), submetidos à hemorroidectomia e complicações pós-operatórias.

\begin{tabular}{lrr}
\hline Informações & $\mathbf{N}$ & \% \\
\hline Total de pacientes atendidos & 34.000 & \\
Portadores de hemorróidas & 9.289 & 27,3 \\
Portadores de DH operados & 2.417 & 26,0 \\
Complicações pós-operatórias & 71 & 3,1 \\
\hline
\end{tabular}


complicações $(3,4 \%)$; e 171 pacientes foram operados por técnica mista, com leitos abertos e fechados $(7,1 \%)$, causando 7 complicações $(4,1 \%)$ (tabela 1 e figuras 1 e 2).

Incidência específica de complicações póshemorroidectomia:

Das 76 complicações pós-hemorroidectomia destacou-se a estenose anal cirúrgica com 44 casos $(1,8 \%)$, seguindo a hemorragia anal cirúrgica com 21 casos $(0,9 \%)$, o agravamento da incontinência anal com 5 casos $(0,2 \%)$, a sepse anal grave com 3 casos $(0,1 \%)$ e 3 casos de complicações clínicas graves $(0,1 \%)-2$ casos de crise hipertensiva com hemorragia controlada clinicamente e com transfusão de sangue e 1 caso de choque anafilático com parada cardíaca e ressuscitação (tabela 2 e figura 3 ).

Incidência absoluta e específica de complicações pós-hemorroidectomia de acordo com as técnicas cirúrgicas empregadas:

A hemorroidectomia aberta, realizada em 2.014 pacientes resultou em 61 complicações $(3,0 \%)$, assim distribuídas: técnica aberta com 2.014 casos e 61 complicações $(1,9 \%)$, das quais 38 casos de estenose anal $(1,9 \%), 19$ casos de hemorragia anal $(0,9 \%), 3$ casos de agravamento de incontinência fecal $(0,1 \%)$, nenhum caso de sepse anal $(0 \%)$ e 1 caso de complicação sistêmica - crise hipertensiva com hemorragia vicariante - $(0,05 \%)$; técnica fechada com 232 casos e 8 complicações $(3,4 \%)$, das quais 4 ca-

Tabela 1 - Complicações pós-hemorroidectomia: distribuição de 2.417 pacientes operados de doença hemorroidária $(\mathrm{DH})$ pelas técnicas cirúrgicas básicas empregadas (aberta, fechada e mista) e incidência de complicações decorrente de cada técnica cirúrgica empregada.

\begin{tabular}{lrrrr}
\hline & \multicolumn{2}{c}{ Cirurgias } & \multicolumn{2}{c}{ Complicações } \\
\cline { 2 - 5 } Técnicas cirúrgicas & $\mathbf{N}$ & $\boldsymbol{\%}$ & $\mathbf{N}$ & \% \\
\hline Aberta (Milligan-Morgan) & 2.014 & 83,3 & 61 & 3,0 \\
Fechada (Ferguson) & 232 & 9,6 & 8 & 3,4 \\
Mista (leitos abertos e fechados) & 171 & 7,1 & 7 & 4,1 \\
Total & $\mathbf{2 . 4 1 7}$ & $\mathbf{1 0 0 , 0}$ & $\mathbf{7 6}$ & $\mathbf{3 , 1}$ \\
\hline
\end{tabular}

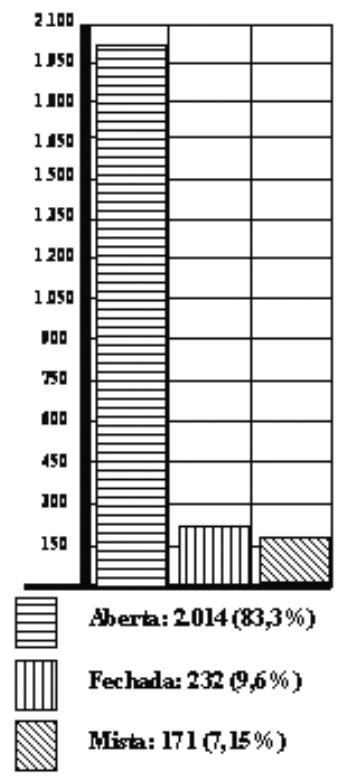

Figura 1 - Complicações pós-hemorroidectomia: técnicas cirúrgicas básicas utilizadas em 2.417 pacientes submetidos à hemorroidectomia (aberta, fechada e mista).

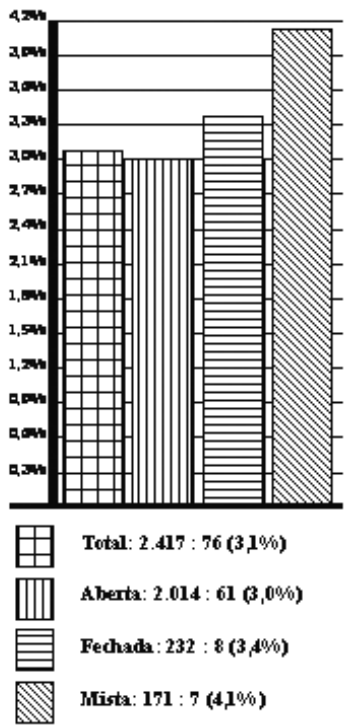

Figura 2 - Complicações pós-hemorroidectomia: incidências, em números percentuais, em termos apenas comparativos, de 76 complicações decorrentes de 2.417 hemorroidectomias (3,1\%), de acordo com as técnicas cirúrgicas utilizadas. 
Tabela 2 - Complicações pós-hemorroidectomia: complicações importantes, imediatas e de longo prazo, decorrentes de 2.417 hemorroidectomias *.

\begin{tabular}{lrc}
\hline Complicações & N & $\mathbf{\%}$ \\
\hline Estenose anal cirúrgica & 44 & $1,8 \%$ \\
Hemorragia anal cirúrgica & 21 & $0,9 \%$ \\
Agravamento da incontinência anal & 5 & $0,2 \%$ \\
Sepse anal grave & 3 & $0,1 \%$ \\
Complicações clínicas graves** & 3 & $0,1 \%$ \\
Total & $\mathbf{7 6}$ & $\mathbf{3 , 1 \%}$ \\
\hline
\end{tabular}

(*) Não foram computadas complicações com as seguintes características: pequena gravidade, resolução clínica e de causas múltiplas - cirúrgicas, anestésicas, medicamentosas, de posição na mesa cirúrgica - como e.g., retenção urinária, cefaléia, lombalgia, febre sem causa aparente, dentre outras.

(**) Crise hipertensiva com hemorragia controlada clinicamente e com transfusão de sangue (2 pacientes); choque anafilático com parada cardíaca e ressuscitação (1 paciente).

sos de estenose anal $(1,7 \%), 1$ caso de hemorragia anal $(0,4 \%), 1$ caso de agravamento de incontinência fecal $(0,4 \%), 2$ casos de sepse anal $(0,9 \%)$ e nenhum caso de complicações sistêmicas $(0 \%)$; técnica mista com 171 casos e 7 complicações $(4,1 \%)$, das quais 2 casos de estenose anal (1,2\%), 1 caso de hemorragia anal $(0,6 \%), 1$ caso de agravamento de incontinência fecal $(0,6 \%), 1$ caso de sepse anal $(0,6 \%)$ e 2 casos de complicações sistêmicas - 1 caso de crise hipertensiva com hemorragia vicariante e 1 caso de choque anafilático com parada cardíaca e ressuscitação - $(1,2 \%)$ (tabela 3 e figura 4$)$.

Incidência absoluta de complicações póshemorroidectomia em 2.417 pacientes operados de acordo com os sexos:

Dos 2.417 pacientes operados de DH, 1.330 eram do sexo feminino $(55,0 \%)$, dos quais 40 desenvolveram complicações pós-hemorroidectomia (3,0\% em relação aos 1.330 operados e $52,6 \%$ dos casos de complicações), e 1.087 do sexo masculino (45,0\%), dos quais 36 desenvolveram complicações póshemorroidectomia (3,3\% em relação aos 1.087 operados e 47,4\% dos casos de complicações) (tabela 4 e figura 5).

Incidências específicas das várias espécies de complicações pós-hemorroidectomia em 2.417 pacientes operados de acordo com os sexos:

Em relação aos 40 casos de complicações desenvolvidas pelas 1.330 mulheres em pós-

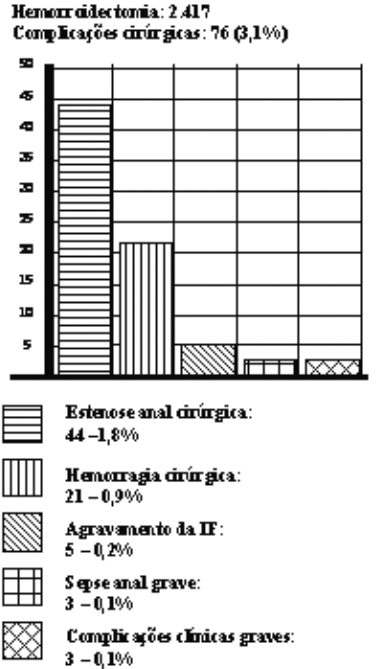

Figura 3 - Complicações pós-hemorroidectomia: complicações decorrentes de 2.417 hemorroidectomias praticadas - estenoses anais cirúrgicas, hemorragias cirúrgicas, agravamento da incontinência fecal parcial, sepse anal grave e complicações clínicas graves.

hemorroidectomia $(3,0 \%)$, sobressaíram as estenoses anais (27 casos, 2,0\%), seguindo a hemorragia anal (7 casos, $0,6 \%)$, o agravamento da incontinência fecal (5 casos, $0,4 \%)$ e a sepse anal ( 1 caso, $0,07 \%$ ) e nenhum caso de complicações sistêmicas $(0 \%)$. E, em relação aos 36 casos de complicações desenvolvidas pelos 1.087 homens em pós-hemorroidectomia $(3,3 \%)$, sobressaíram as estenoses anais (17 casos, 1,6\%), a hemorragia anal (14 casos, 1,3\%), as complicações sistêmicas (3 casos, $0,3 \%)$ e a sepse anal ( 2 casos, $0,2 \%$ ), e nenhum caso de agravamento de incontinência fecal $(0 \%)$ (tabela 5 e figura 6 ).

Incidência absoluta de complicações póshemorroidectomia em 2.417 pacientes operados de acordo com as idades:

$\mathrm{Na}$ segunda década havia 137 pacientes operados $(5,6 \%)$, resultando 2 complicações $(1,5 \%)$, na terceira década havia 502 pacientes operados $(20,8 \%)$ decorrendo 8 complicações $(3,6 \%)$; na quarta década havia 688 pacientes operados $(28,5 \%)$, decorrendo 27 casos de complicações $(3,9 \%)$; na quinta década havia 543 pacientes operados $(22,5 \%)$ decorrendo 14 casos de complicações $(2,6 \%)$, na sexta década havia 274 pacientes operados $(11,3 \%)$ resultando 7 complicações $(2,6 \%)$; na sétima década havia 152 pacientes operados $(6,3 \%)$, resultando 5 casos de complicações $(3,3 \%)$; na oitava década havia 110 pacientes operados $(4,5)$, resultando 3 casos de complicações $(2,7 \%)$; na nona década havia 11 pacientes operados $(0,5)$ sem compli- 
Tabela 3 - Complicações pós-hemorroidectomia: incidência específica de cada complicação pós-operatória em 2.417 pacientes operados de doença hemorroidária $(\mathrm{DH})$, de acordo com as técnicas cirúrgicas praticadas (aberta, fechada e mista), em números absolutos $(N)$ e percentuais (\%).

\begin{tabular}{|c|c|c|c|c|c|c|}
\hline $\begin{array}{l}\text { Técnicas } \\
\text { Cirúrgicas }\end{array}$ & $\begin{array}{c}\text { Complicações } \\
\text { N...........\% }\end{array}$ & $\begin{array}{r}\text { Estenose } \\
\text { N...........\% }\end{array}$ & $\begin{array}{c}\text { Hemorragia } \\
\text { N............\% }\end{array}$ & $\begin{array}{l}\text { Inc. Fecal } \\
\text { N.........\%\% }\end{array}$ & $\begin{array}{c}\text { Sepse } \\
\text { N........\% }\end{array}$ & $\begin{array}{c}\text { Outras* } \\
\text { N..........\% }\end{array}$ \\
\hline Aberta & . 3,0 & .. 1,9 & $19 \ldots \ldots .0,9$ & $3 \ldots \ldots 0,1$ & $\ldots 0,0$ & 0,05 \\
\hline Fechada & $8 \ldots . .3,4$ & $4 \ldots \ldots .1,7$ & $1 \ldots \ldots . .4$ & $1 \ldots \ldots ., 4$ & $2 \ldots \ldots .0,9$ & $0 \ldots \ldots, 0$ \\
\hline Mista & $7 \ldots . .4,1$ & $2 \ldots \ldots .1,2$ & $1 \ldots \ldots ., 6$ & $1 \ldots \ldots 0,6$ & $1 \ldots \ldots .0,6$ & $2 \ldots \ldots 1,2$ \\
\hline Total & $76 \ldots . . .3,1$ & $44 \ldots . . . .1,8$ & $21 \ldots . . . .0,9$ & $5 \ldots . . .0,2$ & $3 \ldots . . .0,1$ & $3 \ldots . . .0,1$ \\
\hline
\end{tabular}

(*) Crise hipertensiva com hemorragia controlada clinicamente e com transfusão de sangue (2 pacientes); choque anafilático com parada cardíaca e ressuscitação (1 paciente).

cações $(0 \%)$ e na décima década não houve pacientes operados (tabela 6 e figura 7).

Incidência específica de complicações póshemorroidectomia em 2.417 pacientes operados de acordo com as idades:

Na primeira faixa etária não existiu pacientes operados; na segunda década houve 137 operados $(5,6 \%)$, com 2 complicações $(1,5 \%)$,ambas sendo estenose anal $(1,5 \%)$; na terceira década houve 502 operados $(20,8 \%)$, com 18 complicações $(3,6 \%)$, sendo 12 casos de estenose anal $(2,4 \%)$ e 6 casos de hemorragia anal (1,2\%); na quarta década houve 688 operados (28,5\%), sendo 27 complicações $(3,9 \%)$, das quais 18 casos eram de estenose anal (2,6\%), 6 casos de hemorragia anal $(0,9 \%), 2$ casos de agravamento da incontinência fecal $(0,3 \%)$ e 1 caso $(0,1 \%)$ de sepse anal $(0,1 \%)$, não tendo havido complicações sistêmicas; na quinta década houve 543 operados $(22,5 \%)$, sendo 14 complicações $(2,6 \%)$, das quais 8 casos eram de estenose anal (1,5\%), 5 casos de hemorragia anal $(0,9 \%), 1$ caso de agravamento de incontinência fecal $(0,2 \%)$ sem qualquer complicação sistêmica; na sexta década houve 274 operados $(11,3 \%)$, sendo 7 complicações $(2,6 \%)$, dos quais 3 casos eram de estenose anal $(1,1 \%), 3$ casos de hemorragia anal $(1,1 \%)$, nenhum caso de agravamento de incontinência anal ou sepse anal $(0 \%)$ e 1 caso de complicação sistêmica $(0,4 \%)$, que foi um caso de crise hipertensiva com hemorragia vicariante; na sétima década houve 152 operados $(6,3 \%)$, sendo 5 complicações $(3,3)$, das quais 1 caso era de estenose anal $(0,7 \%), 1$ caso de agravamento da incontinência fecal $(0,7 \%), 1$ caso de sepse anal $(0,7 \%), 2$ casos de complicações sistêmicas $(1,3)$ sendo um caso de crise hipertensiva com hemorragia vicariante e um caso de choque anafilático com para-

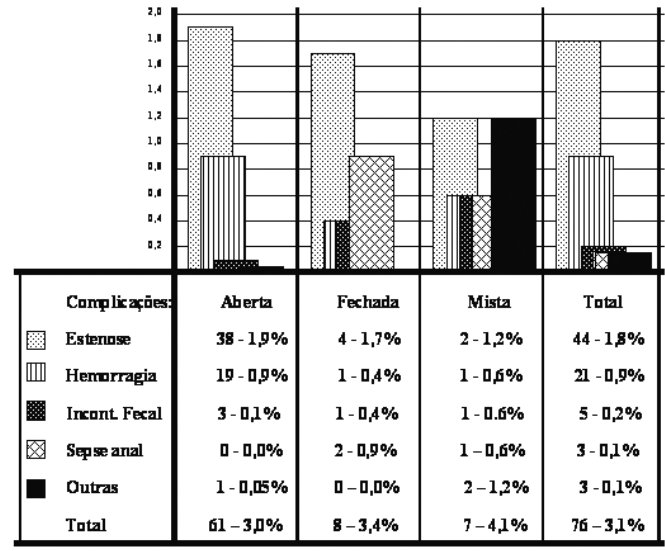

Figura 4 - Complicações pós-hemorroidectomia: gráfico comparando as incidências específicas de cada uma das complicações pós-operatórias em 2.417 pacientes operados de doença hemorroidária $(\mathrm{DH})$, de acordo com as técnicas cirúrgicas praticadas.

da cardíaca e ressuscitação, e nenhum caso de hemorragia anal; na oitava década houve 100 operados $(4,5 \%)$ com 3 complicações $(2,7 \%)$, das quais 1 caso foi de hemorragia anal $(0,9 \%), 1$ caso de agravamento de incontinência anal $(0,9 \%)$ e 1 caso de sepse anal $(0,9 \%)$, e nenhum caso de estenose anal e de complicação sistêmica; na nona década houve 11 operados com nenhuma complicação; e na décima década não houve pacientes operados (tabela 7).

Incidência específica (tônus esfincteriano e fissura anal) de 44 casos de estenose anal póshemorroidectomia de indicação cirúrgica:

Do 44 casos de estenose anal póshemorroidectomia 31 eram sem hipertonia anal $(70,0 \%)$, das quais 23 apresentavam-se com fissura ou área de fibrose $(52,3 \%)$ e 8 sem fissura ou área de fibrose $(17,7 \%)$; e 13 eram com hipertonia anal $(30,0 \%)$, das 
Tabela 4 - Complicações pós-hemorroidectomia: incidência específica de complicações pós-operatórias em 2.417 pacientes operados de doença hemorroidária $(\mathrm{DH})$, de acordo com os sexos, em números absolutos de pacientes operados $(N-1)$ e complicados $(N-2)$ e percentuais de complicações relativas ao número de pacientes de cada sexo operados (\%:N-1) e relativas ao número de complicações de pacientes de cada sexo (\%:N-2).

\begin{tabular}{lccccc}
\hline & \multicolumn{3}{c}{ Operados } & \multicolumn{3}{c}{ Complicados } \\
\hline Sexo & $\mathrm{N}-1$ & $\%$ & $\mathrm{~N}-2$ & $\%: \mathrm{N}-1$ & $\%: \mathrm{N}-2$ \\
Feminino & 1.330 & $55,0 \%$ & 40 & 3,0 & 52,6 \\
Masculino & 1.087 & $45,0 \%$ & 36 & 3,3 & 47,4 \\
Total & 2.417 & $100,0 \%$ & 76 & 3,1 & 100,0 \\
\hline
\end{tabular}

quais 13 apresentavam-se com fissura ou área de fibrose $(6,8 \%)$ e 10 sem área de fissura anal $(23,2 \%)$ (tabela 8).

Incidência específica (forma da estenose anal) $\mathrm{n}$ de 44 casos de estenose anal pós-hemorroidectomia de indicação cirúrgica:

Dos 44 casos de estenose anal póshemorroidectomia 31 tinham a forma anular $(70,4 \%)$, 9 tinham a forma tubular $(20,5 \%)$ e 4 tinham a forma semi-anular $(9,1 \%)$ (tabela 9).

Tempo decorrido entre a hemorroidectomia e o aparecimento de 44 casos de estenose anal póshemorroidectomia:

Em 6 pacientes $(13,5 \%)$ a estenose foi diagnosticada em menos de 10 dias após a cirurgia; em 18 pacientes $(41,0 \%)$ entre 11 e 20 dias; em 9 pacientes $(20,5 \%)$ entre 21 e 30 dias; em 4 pacientes $(9,1 \%)$ entre 30 e 60 dias; e em 7 pacientes $(15,9 \%)$ este dado não foi valorizado (tabela 10).

\section{hemorroidectomia:}

Abordagem cirúrgica da estenose anal pós-

Anotomia simples e excisão de área de fibrose e/ou fissurectomia sem esfincterotomia em 14 (31,8\%) pacientes, com 1 recidiva $(7,1 \%)$; anotomia dupla e excisão de área de fibrose e/ou fissurectomia sem esfincterotomia em $9(20,4 \%)$ pacientes, com 1 recidiva $(11,1 \%)$; anotomia simples e excisão de área de fibrose e/ou fissurectomia com esfincterotomia anal interna (e da porção subcutânea do esfíncter externo) em 6 pacientes $(13,7 \%)$ sem recidiva $(0 \%)$; anotomia dupla e excisão de área de fibrose com esfincterotomia anal interna (e da porção subcutânea do esfíncter externo) em 6 pacientes $(13,7 \%)$, sem recidivas $(0 \%)$; anoplastia com retalho cutâneo em "V" em 7 pacientes, $(15,9 \%)$, com 1 recidiva $(14,3 \%)$; anoplastia com

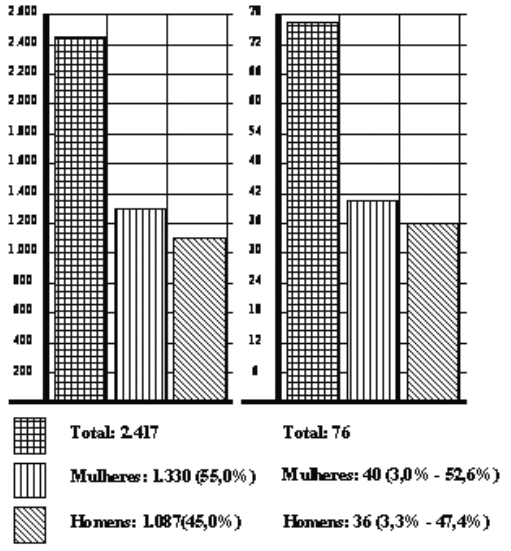

Figura 5 - Complicações pós-hemorroidectomia: a figura da esquerda representa, em números absolutos e percentuais, a incidência de hemorroidectomia (coluna quadriculada) em mulheres (linhas verticais) e homens (linhas diagonais); e figura da direita representa, em números absolutos e percentuais, a incidência de complicações pós-hemorroidectomia (coluna quadriculada) em mulheres (linhas verticais) e homens (linhas diagonais).

retalho cutâneo retangular em 2 pacientes (4,5\%), sem recidivas; totalizando 44 pacientes operados de estenose anal com 3 recidivas $(6,8 \%)$ (tabela 11$)$.

Incidência e abordagem da hemorragia póshemorroidectomia em 21 pacientes operados:

Todos os 21 pacientes que desenvolveram hemorragia anal pós-hemorroidectomia $(0,9 \%)$ foram submetidos à revisão cirúrgica com ligadura e transfixação de pedículo (tabela 12).

Incidência e abordagem do agravamento da incontinência fecal pós-hemorroidectomia em 5 pacientes operados:

Dos 5 pacientes que desenvolveram agravamento da incontinência fecal pós-hemorroidectomia $(0,2 \%), 2$ foram submetidos à esfincteroplastia $(40,0 \%)$ e 3 não foram operados (tabela 13). 
Tabela 5 - Complicações pós-hemorroidectomia: incidências específicas das várias espécies de complicações pós-operatórias em 2.417 pacientes operados de doença hemorroidária (DH), de acordo com os sexos, em números absolutos de pacientes operados.

\begin{tabular}{|c|c|c|c|c|c|c|c|}
\hline Sexos & Cirurgias & Complicações & Estenose & Hemorragia & Inc.Fecal & Sepse & Outras \\
\hline Feminino & 1.330 & $\begin{array}{l}40 \\
3,0 \%\end{array}$ & $\begin{array}{l}27 \\
2,0 \%\end{array}$ & $\begin{array}{l}7 \\
0,5 \%\end{array}$ & $\begin{array}{l}5 \\
0,4 \%\end{array}$ & $\begin{array}{l}1 \\
0,07 \%\end{array}$ & $\begin{array}{l}0 \\
0,0 \%\end{array}$ \\
\hline Masculino & 1.087 & $\begin{array}{l}36 \\
3,3 \%\end{array}$ & $\begin{array}{l}17 \\
1,6 \%\end{array}$ & $\begin{array}{l}14 \\
1,3 \%\end{array}$ & $\begin{array}{l}0 \\
0,0 \%\end{array}$ & $\begin{array}{l}2 \\
0,2 \%\end{array}$ & $\begin{array}{l}3 \\
0,3 \%\end{array}$ \\
\hline Total & 2.417 & $\begin{array}{l}76 \\
3,1 \%\end{array}$ & $\begin{array}{l}44 \\
1,8 \%\end{array}$ & $\begin{array}{l}21 \\
0,9 \%\end{array}$ & $\begin{array}{l}5 \\
0,2 \%\end{array}$ & $\begin{array}{l}3 \\
0,1 \%\end{array}$ & $\begin{array}{l}3 \\
0,1 \%\end{array}$ \\
\hline
\end{tabular}

Incidência e abordagem da sepse anal póshemorroidectomia em 3 pacientes operados:

Todos os 3 pacientes que desenvolveram sepse anal pós-hemorroidectomia foram levados à cirurgia para drenagem e desbridamento, com sucesso (tabela 14).

\section{DISCUSSÃO}

Incidência absoluta de hemorróidas, hemorroidectomias e complicações póshemorroidectomia: há muita controvérsia sobre a epidemiologia da $\mathrm{DH}$, e trabalhos, os mais diversos, têm sido publicados com referência ao estudo da incidência de DH na população, e os dados têm sido conflitantes, com autores estabelecendo incidências médias que vão de próximos a 4,4\%, como Johanson et al ${ }^{23}$; a $86 \%$ da humanidade, conforme afirmado por Hass et al ${ }^{19}$; e passando por incidências em torno de $10 \%$, como admitem Ganchrow et al. ${ }^{17}$ e Iwagaki et al. ${ }^{22}$. Isto faz concluir existência de metodologias completamente diversas, e que pode eivar de erros conclusões precipitadas, pois vão desde a denominação para a DH sintomática até achados de necrópsias. Em estudo comparativo entre grupos sintomáticos e assintomáticos, Hass et al. ${ }^{19}$ relatam uma incidência bastante semelhante, com identificação de hemorróidas em $88 \%$ no grupo sintomático (DH), e $82 \%$ no grupo assintomático (submetido a exame proctológico de rotina para rastreamento de câncer colorretal ou por solicitação de outras clínicas).

Dos 9.289 pacientes portadores de $\mathrm{DH}$ em 34.000 pacientes submetidos ao exame proctológico $(27,3 \%), 2.417(26,0 \%)$ foram submetidos à hemorroidectomia, decorrendo 76 complicações $(3,1 \%)$ (tabela A).

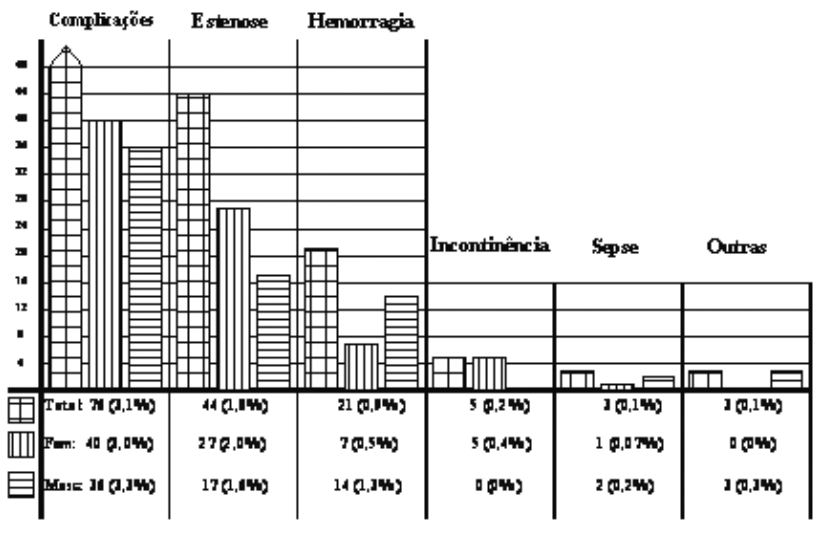

Figura 6 - Complicações pós-hemorroidectomia: figura representativa de 6 conjunto de 3 colunas, representando o total e e o percentual de cada uma das complicações; as colunas representam o total e o percentual de cada complicação em ambos os sexos (coluna quadriculada), em mulheres (linhas verticais) e homens (linhas diagonais).

Incidência absoluta de hemorroidectomia e de complicações pós-hemorroidectomia de acordo com as técnicas cirúrgicas empregadas (aberta, fechada e mista): dos 2.417 pacientes submetidos à hemorroidectomia resultaram 76 complicações (3,1\%): 2.014 pacientes foram operados por técnica aberta ou Milligan-Morgan ${ }^{29}(83,3 \%)$, levando a 61 casos de complicações (3,0\%); 232 pacientes foram operados pela técnica fechada ou de Ferguson ${ }^{13}(9,6 \%)$, levando a 8 complicações $(3,4 \%)$; e 171 pacientes foram operados por técnica mista, com leitos abertos e fechados $(7,1 \%)$, causando 7 complicações $(4,1 \%)$ (tabela 1 e figuras 1 e 2 ).

Ganchrow et al. ${ }^{17}$, em sua série de 2.038 pacientes operados agrupam as complicações pós-operatórias em imediatas (82 casos, 4,0\%) e tardias (99 casos, $4,8 \%$ ), contabilizando complicações sistêmicas, 
Tabela 6 - Complicações pós-hemorroidectomia: incidência global de complicações pós-operatórias em 2.417 pacientes operados de doença hemorroidária (DH), de acordo com as faixas etárias, em números absolutos de pacientes operados.

\begin{tabular}{ccccc}
\hline Faixas etárias & N de DH & \% de DH & N de complicações & \% de complicações \\
\hline $1-10$ & & & 0 & 0,0 \\
$11-20$ & 137 & 5,6 & 02 & 1,5 \\
$21-30$ & 502 & 20,8 & 18 & 3,6 \\
$31-40$ & 688 & 28,5 & 27 & 3,9 \\
$41-50$ & 543 & 22,5 & 14 & 2,6 \\
$51-60$ & 274 & 11,3 & 07 & 2,6 \\
$61-70$ & 152 & 6,3 & 05 & 3,3 \\
$71-80$ & 110 & 4,5 & 03 & 2,7 \\
$81-90$ & 011 & 0,5 & 0 & 0,0 \\
$91-100$ & 000 & 0,0 & 0 & 0,0 \\
Total & 2.417 & 100 & 76 & 3,1 \\
\hline
\end{tabular}

desde pneumonias até cistites. Atendo-se apenas às complicações anais, as complicações de sua série totalizam $127(6,2 \%)$.

Comparando-se nossa série (2.417 pacientes operados, 2.014 por técnica aberta) (tabela 1 e figuras 1 e 2), e os 2.038 da série de Ganchrow et al. ${ }^{17}$, (todos por técnica fechada), verificamos que nosso índice de complicações foi exatamente a metade (76 casos, $3,1 \%$ ) dos da série dos referidos autores (127 casos, $6,2 \%)$.

Incidência específica de complicações pós-hemorroidectomia: em nossa série de 76 complicações pós-hemorroidectomia destacou-se a estenose anal cirúrgica com 44 casos $(1,8 \%)$, seguindo a hemorragia anal cirúrgica com 21 casos $(0,9 \%)$, o agravamento da incontinência anal com 5 casos $(0,2 \%)$, a sepse anal grave com 3 casos $(0,1 \%)$ e 3 casos de complicações clínicas graves $(0,1 \%)-2$ casos de crise hipertensiva com hemorragia controlada clinicamente e com transfusão de sangue e 1 caso de choque anafilático com parada cardíaca e ressuscitação (tabela 2 e figura 3 ).

Ganchrow et al. ${ }^{17}$,em sua série de 2.038 pacientes operados agrupam as complicações pós-operatórias em imediatas ( 82 casos, 4,0\%) e tardias ( 99 casos, $4,8 \%$ ), contabilizando complicações sistêmicas, desde pneumonias até cistites. Atendo-se apenas às complicações anais foram as seguintes as incidências: fissura anal (54 casos, 2,7\%), estenose anal (24 casos, $1,2 \%)$, hemorragia cirúrgica 27 casos $(1,3 \%)$,

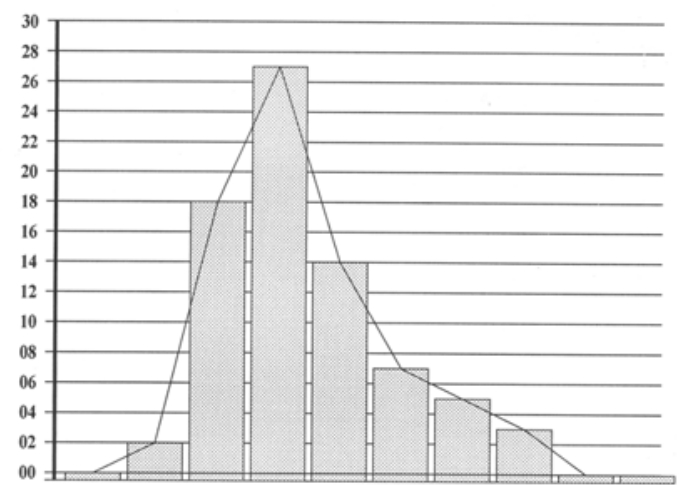

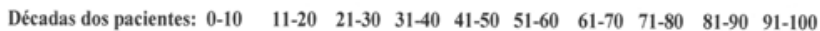
$\begin{array}{lllllllllll}\mathrm{N} \text { de cirurgias (2.417): } & 0 & 137 & 502 & 688 & 543 & 274 & 152 & 110 & 011 & 0\end{array}$ $\mathrm{N}$
$\mathrm{de}$ $\begin{array}{lllllllllll}\text { \% complicações (3,1\%): } & 0 & 1,5 & 3,6 & 3,9 & 2,6 & 2,6 & 3,3 & 2,7 & 0 & 0\end{array}$

Figura 7 - Complicações pós-hemorroidectomia: figura com 10 colunas com listras transversais representando as décadas etárias em que ocorreram as complicações, sendo os totais de cada década unidos por uma linha cheia.

abscesso anal (17 casos, $0,8 \%$ ), fístula anal (5 casos, $0,2 \%)$, totalizando 127 complicações anais $(6,2 \%)$.

Comparando nossa série com a de Ganchrow et al ${ }^{17}$, verificamos as seguintes correspondências das várias complicações: estenoses anais (44 casos, 1,8\% para 24 casos, 1,2\%), hemorragia anal cirúrgica (21 casos, $0,9 \%$ para 27 casos, $1,3 \%$ ), agravamento da incontinência anal (5 casos, $0,2 \%$ contra nenhum caso), abscessos anais ( 3 casos, $0,1 \%$ ) contra 17 casos, $0,8 \%$ ), 
Tabela 7 - Complicações pós-hemorroidectomia: incidências específicas das várias espécies de complicações pós-operatórias em 2.417 pacientes operados de doença hemorroidária (DH), de acordo com as faixas etárias, em números absolutos de pacientes operados.

\begin{tabular}{|c|c|c|c|c|c|c|c|c|}
\hline & $\begin{array}{l}\text { Faixas } \\
\text { etárias }\end{array}$ & $\begin{array}{c}\text { Hemorroi- } \\
\text { dectomia }\end{array}$ & $\begin{array}{l}\text { Complica- } \\
\text { ções }\end{array}$ & $\begin{array}{c}\text { Estenose } \\
\text { anal }\end{array}$ & $\begin{array}{c}\text { Hemorragia } \\
\text { anal }\end{array}$ & $\begin{array}{c}\text { Inconti- } \\
\text { nência }\end{array}$ & $\begin{array}{l}\text { Sepse } \\
\text { anal }\end{array}$ & $\begin{array}{c}\text { Compl. } \\
\text { sistêmicas }\end{array}$ \\
\hline $\mathrm{N}-\%$ & $1-10$ & & & & & & & \\
\hline $\mathrm{N}-\%$ & $11-20$ & $137-\quad 5,6$ & $02-1,5$ & $02-1,5$ & & & & \\
\hline $\mathrm{N}-\%$ & $21-30$ & $502-20,8$ & $18-3,6$ & $12-2,4$ & $06-1,2$ & & & \\
\hline $\mathrm{N}-\%$ & $31-40$ & $688-28,5$ & $27-3,9$ & $18-2,6$ & $06-\quad 0,9$ & $02-0,3$ & $01-0,1$ & \\
\hline $\mathrm{N}-\%$ & $41-50$ & $543-22,5$ & $14-2,6$ & $08-1,5$ & $05-0,9$ & $01-0,2$ & & \\
\hline $\mathrm{N}-\%$ & $51-60$ & $274-11,3$ & $07-2,6$ & $03-1,1$ & $03-1,1$ & & & $01-0,4$ \\
\hline $\mathrm{N}-\%$ & $61-70$ & $152-\quad 6,3$ & $05-3,3$ & $01-0,7$ & & $01-0,7$ & $01-0,7$ & $02-1,3$ \\
\hline $\mathrm{N}-\%$ & $71-80$ & $110-\quad 4,5$ & $03-2,7$ & & $01-0,9$ & $01-0,9$ & $01-0,9$ & \\
\hline $\mathrm{N}-\%$ & $81-90$ & $011-\quad 0,5$ & & & & & & \\
\hline $\mathrm{N}-\%$ & $91-100$ & $000-\quad 0,0$ & & & & & & \\
\hline $\mathrm{N}-\%$ & Total & $2.417-100$ & $76-3,1$ & $44-1,8$ & $21-0,9$ & $5-0,2$ & $3-0,1$ & $3-0,1$ \\
\hline
\end{tabular}

fissuras anais (nenhum caso para 54 casos, 2,7\%) e fístulas anais (nenhum caso para 5 casos, $0,2 \%$ ).

Incidência absoluta e específica de complicações pós-hemorroidectomia de acordo com as técnicas cirúrgicas empregadas: como se pode constatar analisando a tabela 3 e a figura 4 , não houve diferença estatisticamente significativa entre as complicações pelas três técnicas usadas: hemorroidectomia aberta em 2.014 pacientes resultou em 61 complicações $(3,0 \%)$; hemorroidectomia fechada em 232 pacientes resultou em 8 complicações $(3,4 \%)$; e hemorroidectomia mista em 171 pacientes resultou em 7 complicações $(4,1 \%)$, com média geral de $3,1 \%$.
Dos 44 casos de estenose anal $38(1,9 \%)$ ocorreram em hemorroidectomia aberta, 4 em hemorroidectomia fechada $(1,7 \%)$ e 2 em hemorroidectomia mista $(1,2 \%)$. Dos 21 casos de hemorragia anal cirúrgica, 19 ocorreram em hemorroidectomia aberta $(0,9 \%), 1$ em hemorroidectomia fechada $(0,4 \%)$ e 1 em hemorroidectomia mista $(0,6 \%)$. Dos 5 casos de agravamento de incontinência fecal, 3 ocorreram em hemorroidectomia aberta $(0,1 \%), 1$ em hemorroidectomia fechada $(0,4 \%)$ e 1 em hemorroidectomia mista $(0,6 \%)$. Dos 3 casos de abscesso anal, nenhum ocorreu em hemorroidectomia aberta (0\%), 2 em hemorroidectomia fechada $(0,9 \%)$ e $1 \mathrm{em}$

Tabela 8 - Complicações pós-hemorroidectomia: abordagem da estenose anal pós hemorroidectomia de indicação cirúrgica (EAPHIC) - resultados de exame proctológico em 44 pacientes portadores de EAPHIC, em relação à concomitância de hipertonia e de fissura anal.

\begin{tabular}{lcc}
\hline Estenose Anal & N & \% \\
\hline Com Hipertonia Anal e sem Fissura Anal & 03 & $6,8 \%$ \\
Com Hipertonia Anal e com Fissura Anal & 10 & $23,2 \%$ \\
Com Hipertonia Anal & 13 & $30,0 \%$ \\
& & \\
Sem Hipertonia Anal e com Fissura Anal & 23 & $52,3 \%$ \\
Sem Hipertonia Anal e sem Fissura Anal & 08 & $17,7 \%$ \\
Sem Hipertonia Anal & 31 & $70,0 \%$ \\
& & $100,0 \%$ \\
\hline
\end{tabular}


Tabela 9 - Complicações pós-hemorroidectomia: abordagem da estenose anal pós hemorroidectomia de indicação cirúrgica (EAPHIC) - resultados de exame proctológico em 44 pacientes portadores de EAPHIC, em relação ao formato da estenose e do canal anal.

\begin{tabular}{lcc}
\hline Forma da Estenose Anal & $\mathbf{N}$ & $\mathbf{\%}$ \\
\hline Semi-anulares & 04 & $9,1 \%$ \\
Anulares & 31 & $70,4 \%$ \\
Tubulares & 09 & $20,5 \%$ \\
Total & 44 & $100,0 \%$ \\
\hline
\end{tabular}

Tabela 10 - Complicações pós-hemorroidectomia: abordagem da estenose anal pós hemorroidectomia de indicação cirúrgica (EAPHIC) - tempo decorrido entre a hemorroidectomia e o diagnóstico definitivo da EAPHIC.

\begin{tabular}{llr}
\hline Tempo decorrido & $\mathbf{N}$ & $\mathbf{\%}$ \\
\hline Menos de 10 dias & 06 & $13,5 \%$ \\
11 - 20 dias & 18 & $41,0 \%$ \\
21 - 30 dias & 09 & $20,5 \%$ \\
30 dias - 60 dias & 04 & $9,1 \%$ \\
Ignorado & 07 & $15,9 \%$ \\
Total & 44 & $100,0 \%$ \\
\hline
\end{tabular}

hemorroidectomia mista $(0,6 \%)$. E dos 3 casos de complicações sistêmicas, 1 ocorreu em hemorroidectomia aberta $(0,05 \%)$, nenhum em hemorroidectomia fechada $(0 \%)$ e 2 em hemorroidectomia mista $(1,2 \%)$ (tabela 3 e figura 4). Como não houve diferença estatisticamente significativa entre as várias técnicas cirúrgicas usadas e as complicações pós-operatórias torna-se desnecessário compararem-se, especificamente, os achados da série de Ganchrow et al. ${ }^{17}$ (somente técnica fechada de Ferguson) e os nossos 232 pacientes submetidos à hemorroidectomia por técnica fechada.

Incidência absoluta de hemorroidectomia e de complicações pós-hemorroidectomia em 2.417 pacientes operados de acordo com os sexos: a maioria dos autores admite predominância da DH no homem, às vezes com uma freqüência quase em dobro em relação às mulheres. Santos ${ }^{44,45}$, em sua experiência pessoal com 1.327 pacientes operados de $\mathrm{DH}$, encontrou 768 homens $(57,9 \%)$ e 559 mulheres $(42,13 \%)$. Corroborando estes achados, Ganchrow et al. ${ }^{17} \mathrm{em}$ série de 2.038 pacientes operados de $\mathrm{DH}$ por técnica fechada de Ferguson, encontraram 1.237 homens $(62,7 \%)$ e 761 mulheres $(37,3 \%)$.

Contrariamente à maioria dos autores, mas ressaltando-se as séries de Ganchrow et al. ${ }^{17}(62,7 \%$ de homens) e de $\operatorname{Santos}^{44,45}$ (57,9\% de homens), tanto a DH (5.007 mulheres, 53,9\%; 4.282 homens, $46,1 \%)$

Tabela 11 - Complicações pós-hemorroidectomia: abordagem da estenose anal pós hemorroidectomia de indicação cirúrgica (EAPHIC) - técnicas cirúrgicas empregadas na abordagem de 44 pacientes portadores de (EAPHIC) e recidivas.

\begin{tabular}{|c|c|c|c|}
\hline Técnica Cirúrgica & $\mathbf{N}$ & $\%$ & Recidiva \\
\hline $\begin{array}{l}\text { Anotomia simples e excisão de área de fibrose e/ou } \\
\text { fissurectomia sem esfincterotomia }\end{array}$ & 14 & $31,8 \%$ & $1(7,1 \%)$ \\
\hline $\begin{array}{l}\text { Anotomia dupla e excisão de área de fibrose e/ou } \\
\text { fissurectomia sem esfincterotomia }\end{array}$ & 09 & $20,4 \%$ & $1(11,1 \%)$ \\
\hline $\begin{array}{l}\text { Anotomia simples e excisão de área de fibrose e/ou fissurectomia } \\
\text { com esfincterotomia anal interna (e da porção subcutânea } \\
\text { do esfíncter externo) }\end{array}$ & 06 & $13,7 \%$ & $0 \quad(0 \%)$ \\
\hline $\begin{array}{l}\text { Anotomia dupla e excisão de área de fibrose com esfincterotomia } \\
\text { anal interna (e da porção subcutânea do esfíncter externo) }\end{array}$ & 06 & $13,7 \%$ & $0 \quad(0 \%)$ \\
\hline Anoplastia com retalho cutâneo em "V" & 07 & $15,9 \%$ & $1(14,3 \%)$ \\
\hline Anoplastia com retalho cutâneo retangular & 02 & $4,5 \%$ & $0 \quad(0 \%)$ \\
\hline Anoplastia com retalho mucoso, ou abaixamento mucoso & 0 & $0,0 \%$ & \\
\hline $\begin{array}{l}\text { Anoplastia com duplo retalho ou retalho misto ou retalhos } \\
\text { cutâneo e mucoso }\end{array}$ & 0 & $0,0 \%$ & \\
\hline Total & 44 & $100,0 \%$ & $3(6,8 \%)$ \\
\hline
\end{tabular}


Tabela 12 - Complicações pós-hemorroidectomia: abordagem da hemorragia anal pós-hemorroidectomia de indicação cirúrgica (HAPHIC) - técnicas cirúrgicas empregadas na abordagem de 21 pacientes portadores de HAPHIC.

\begin{tabular}{cccc}
\hline N de cirurgias para DH & N HAPHIC & \%HAPHIC & Transfixação dos pedículos \\
\hline 2.417 & 21 & $0,9 \%$ & $21-100.0 \%$ \\
\hline
\end{tabular}

Tabela 13 - Complicações pós-hemorroidectomia: abordagem do agravamento da incontinência fecal póshemorroidectomia de indicação cirúrgica (AIFPH) - esfincteroplastia "em jaquetão" - cirurgias empregadas na abordagem de 2 de 5 pacientes com AIFPH.

\begin{tabular}{ccccc}
\hline N de cirurgias para DH & N AIFPH & \% AIFPH & Cirurgias Corretivas & Não Operados \\
\hline 2.417 & 5 & $0,2 \%$ & $2-40,0 \%$ & $3-60,0 \%$ \\
\hline
\end{tabular}

Tabela 14 - Complicações pós-hemorroidectomia: abordagem da sepse anal pós-hemorroidectomia de indicação cirúrgica (SAPHIC) - drenagem - cirurgia empregada na abordagem dos 3 pacientes com SAPHIC.

\begin{tabular}{cccc}
\hline N de cirurgias para DH & N SAPHIC & \% SAPHIC & Drenagem \\
\hline 2.417 & 3 & $0,1 \%$ & $3-100,0 \%$ \\
\hline
\end{tabular}

(tabela 4 e figura 5) quanto as hemorroidectomias (1.330 ou $55,0 \%$ dos 2.417 operados) foram mais comum entre mulheres, de forma estatisticamente significativa.

Incidência específica das várias espécies de complicações pós-hemorroidectomia em 2.417 pacientes operados de acordo com os sexos:

Das 40 mulheres que desenvolveram complicações pós-hemorroidectomia $(3,0 \%)$ sobressaíram as estenoses anais ( 27 casos, 2,0\%), a hemorragia anal ( 7 casos, $0,6 \%$ ), o agravamento da incontinência fecal ( 5 casos, $0,4 \%)$ e a sepse anal ( 1 caso, $0,07 \%)$ e nenhum caso de complicações sistêmicas (0\%); e dos 36 homens que desenvolveram complicações póshemorroidectomia (3,3\%), sobressaíram as estenoses anais (17 casos, 1,6\%), a hemorragia anal (14 casos, $1,3 \%$ ), as complicações sistêmicas ( 3 casos, $0,3 \%$ ) e a sepse anal ( 2 casos, $0,2 \%$ ), e nenhum caso de agravamento de incontinência fecal $(0 \%)$ (tabela 5 e figura 6).

Nota-se que as mulheres desenvolveram mais estenoses (27 casos, 2,0\% contra 17 casos, $1,6 \%)$ e agravamento da incontinência fecal (5 casos, 0,4\% contra nenhum caso, $0 \%$ ), e os homens desenvolve- ram mais hemorragia cirúrgica ( 14 casos, $1,3 \%$ contra 7 casos, $0,6 \%$ ), sepse anal ( 2 casos, $0,2 \%$ contra 1 caso, $0,07 \%$ ) e complicações sistêmicas ( 3 casos, $0,3 \%$ contra nenhum, $0 \%$ ) (tabela 5 e figura 6 ).

Não houve como aferir estes dados com a literatura, pois a diferença de complicações póshemorroidectomia por sexos não foi privilegiada pelos autores consultados.

Incidência absoluta de complicações póshemorroidectomia em 2.417 pacientes operados de acordo com as idades: são raríssimas as ocorrências descritas de DH na infância e muito pouco freqüentes antes dos 20 anos, admitindo alguns autores incidências mais elevadas entre 30 e 60 anos. Após os 60 anos, com a diminuição de alguns fatores desencadeantes, a incidência decresce, cedendo lugar ao aparecimento de doenças relacionadas à diminuição do tônus muscular pelviperineal e do assoalho pélvico, tendo Santos ${ }^{44,45}$, em 1.327 portadores de DH encontrado $53,9 \%$ dos casos em pacientes entre $31 \mathrm{e}$ 50 anos e 70,0\% entre 31 e 60 anos. Ganchrow et al. ${ }^{17}$, em série de 2.038 pacientes operados de $\mathrm{DH}$ encontrou apenas 37 pacientes com idades inferiores a 20 
anos (1,8\%), e 666 pacientes com idades entre 21 e 39 anos $(32,7 \%)$ e 1.035 entre 40 e 59 anos $(50,8 \%)$.

A média etária dos pacientes por ocasião do diagnóstico, em nossa série, foi de 39,9 anos, com 51,4\% dos pacientes com idades entre 31 e 50 anos e $74,8 \%$ entre 21 e 50 anos; e a média etária dos pacientes por ocasião da cirurgia foi de 41,6 anos, com $51,0 \%$ com idades entre 31 e 40 anos e $71,8 \%$ entre 21 e 50 anos (tabela 6 e figura 7). Conclui-se, portanto, que em nossa série, as idades dos pacientes, tanto por ocasião do diagnóstico quanto por ocasião da cirurgia foram muito inferiores às séries relatadas 17,30 .

Das 76 complicações, 44 foram estenoses anais $(1,8 \%), 21$ foram hemorragias anais cirúrgicas $(0,9 \%)$, 5 foram agravamento da incontinência fecal $(0,2 \%), 3$ foram sepse anal (abscessos) $(0,1 \%)$ e 3 foram complicações sistêmicas $(0,1 \%)$ (tabela 7$)$. As faixas etárias em que mais ocorreram estenoses anais (44 casos, $1,8 \%)$ foram a quarta (18 casos, $2,6 \%)$ e a terceira ( 12 casos, 2,4\%); não houve estenoses nas 3 últimas décadas. A hemorragia anal (21 casos, $0,9 \%$ ) ocorreu mais na terceira ( 6 casos, $1,2 \%$ ) e na sexta ( 3 casos, $1,1 \%$ ) décadas, não tendo ocorrido na sétima, e nas duas últimas décadas. $\mathrm{O}$ agravamento da incontinência anal (5 casos, $0,2 \%$ ) ocorreu mais na oitava década ( 1 caso em 110 operados, $0,9 \%$ ) e na sétima ( 1 caso em 152 operados, $0,7 \%$ ) décadas, sem ocorrência nas duas primeiras, na sexta e nas duas últimas décadas. A sepse anal (3 casos, $0,1 \%$ ) ocorreu mais na oitava (1 caso em 110 cirurgias, $0,9 \%$ ) e na sétima ( 1 caso em 152 cirurgias, $0,7 \%$ ), sem ocorrência nas segunda, terceira, quinta, sexta e as duas últimas décadas. Já as 3 complicações sistêmicas $(0,1 \%)$ ocorreram na sétima ( 2 casos em 152 cirurgias, 1,3\%) e na sexta (1 caso em 274 cirurgias, 0,4\%) décadas (tabela 7). Não houve como aferir estes dados com a literatura, pois a diferença de complicações pós-hemorroidectomia por sexos não foi privilegiada pelos autores consultados.

Incidência absoluta de estenose anal de indicação cirúrgica como complicação póshemorroidectomia: em nossa série de 76 complicações em 2.417 pacientes submetidos à hemorroidectomia $(3,1 \%)$ as estenoses anais foram as mais comuns, com 44 casos $(1,8 \%)$ (tabela 7$)$. Na série de Ganchrow et al. ${ }^{14}$ de 127 complicações anais pós-operatórias em 2.038 pacientes operados de $\mathrm{DH}$ $(6,2 \%)$ as estenoses anais ficaram em segundo lugar com 24 casos $(1,2 \%)$. Todavia ele relata uma incidência de 54 casos de fissura anal $(2,7 \%)$. Se somar- mos fissuras anais e estenose anal (pois em nossa série não contabilizamos fissura anal como a complicação primária), a incidência de estenose anal passa para 78 casos $(3,9 \%)$, portanto maior que nossa incidência $(2,7 \%)$. Se compararmos os 2.038 casos de Ganchrow et al. ${ }^{14}$ (todos operados por técnica fechada) os 232 pacientes de nossa série operados apenas por esta técnica, verificamos que ocorreram 4 casos de estenose anal $(1,7 \%)$, portanto uma incidência menor que a de Ganchrow et al. ${ }^{14}(3,9 \%)$. Uma possível explicação para a diferença marcante entre nossa incidência de estenose anal $(1,8 \%)$ e a apresentada por Ganchrow et al. ${ }^{14}(3,9 \%)$ é o fato de termos somente contabilizado casos de estenose anal de indicação cirúrgica e aquele autor poder ter incluído tanto casos de indicação cirúrgica quanto casos de estenose anal resolvidos com simples dilatações anais digitais ou com velas de Hegar.

Incidência específica (tônus esfincteriano e fissura anal) de $\mathbf{4 4}$ casos de estenose anal póshemorroidectomia de indicação cirúrgica: dos 44 casos de estenose anal pós-hemorroidectomia, 31 eram sem hipertonia anal $(70,0 \%)$, das quais 23 apresentavam-se com fissura ou área de fibrose $(52,3 \%)$ e 8 sem fissura ou área de fibrose (17,7\%); e 13 eram com hipertonia anal (30,0\%), das quais 13 apresentavam-se com fissura ou área de fibrose $(6,8 \%)$ e 10 sem área de fissura anal $(23,2 \%)$ (tabela 8$)$. Isto mostra, claramente, que a estenose anal nada tem a ver com o fato de o paciente ser ou não portador de hipertonia anal. A estenose é um problema relacionado, não ao tônus esfincteriano, mas a um defeito de cicatrização, seja ele oriundo da própria técnica cirúrgica, seja decorrente do próprio paciente. Não houve como aferir estes dados com a literatura, pois a diferença de complicações pós-hemorroidectomia por sexos não foi privilegiada pelos autores consultados.

Incidência específica (forma da estenose anal) de 44 casos de estenose anal póshemorroidectomia de indicação cirúrgica: dos 44 casos de estenose anal pós-hemorroidectomia, 31 tinham a forma anular $(70,4 \%), 9$ tinham a forma tubular $(20,5 \%)$ e 4 tinham a forma semi-anular $(9,1 \%)$ (tabela 9). Isto deixa claro que a indicação cirúrgica mais evidente é a própria anotomia, ou seja, seccionar, em um ou mais pontos, o anel fibroso. Não houve como aferir estes dados com a literatura, pois a diferença de complicações pós-hemorroidectomia por sexos não foi privilegiada pelos autores consultados. 
Tempo decorrido entre a hemorroidectomia e o aparecimento de 44 casos de estenose anal pós-hemorroidectomia: em 6 pacientes $(13,5 \%)$ a estenose foi diagnosticada em menos de 10 dias após a cirurgia; em 18 pacientes $(41,0 \%)$ entre 11 e 20 dias; em 9 pacientes $(20,5 \%)$ entre 21 e 30 dias; em 4 pacientes $(9,1 \%)$ entre 30 e 60 dias; e em 7 pacientes $(15,9 \%)$ este dado não foi valorizado (tabela 10). É muito importante o fato de que a estenose anal já ocorra pouco tempo depois da cirurgia (75,0\% antes de decorrido um mês), período em que o cirurgião ainda está acompanhando o paciente e, portanto, ainda em tempo de se obter um bom resultado com dilatação manual e/ou instrumental do canal anal. Não houve como aferir estes dados com a literatura, pois a diferença de complicações póshemorroidectomia por sexos não foi privilegiada pelos autores consultados.

Abordagem cirúrgica da estenose anal pós hemorroidectomia: como dos 44 casos de estenose anal pós-hemorroidectomia a maioria tinha a forma anular (31 casos, 70,4\%), a cirurgia mais praticada foi a anotomia ( 35 casos, $79,6 \%$ ), em suas mais variadas formas e composições: (a) anotomia simples e excisão de área de fibrose e/ou fissurectomia sem esfincterotomia em 14 (31,8\%), (b) anotomia dupla e excisão de área de fibrose e/ou fissurectomia sem esfincterotomia em $9(20,4 \%)$ pacientes, (c) anotomia simples e excisão de área de fibrose e/ou fissurectomia com esfincterotomia anal interna (e da porção subcutânea do esfíncter externo) em 6 pacientes (13,7\%); e (d) e anotomia dupla e excisão de área de fibrose com esfincterotomia anal interna (e da porção subcutânea do esfíncter externo) em 6 pacientes $(13,7 \%)$.As demais técnicas dependeram das formas das estenoses (9 tinham a forma tubular, 20,5\% e 4 tinham a forma semi-anular, 9,1\%): anoplastia com retalho cutâneo em "V" em 7 pacientes $(15,9 \%)$ e anoplastia com retalho cutâneo retangular em 2 pacientes (4,5\%). Mas nem sempre as cirurgias corretivas de estenose anal resultaram em sucesso: houve 3 recidivas $(6,8 \%)$. As cirurgias que resultaram em recidivas foram: (a) anotomia simples e excisão de área de fibrose e/ou fissurectomia sem esfincterotomia com 1 recidiva $(7,1 \%)$; (b) anotomia dupla e excisão de área de fibrose e/ou fissurectomia sem esfincterotomia com 1 recidiva $(11,1 \%)$; e (c) anoplastia com retalho cutâneo em "V" com 1 recidiva $(14,3 \%)$; totalizando 44 pacientes operados de estenose anal com 3 recidivas $(6,8 \%)$ (tabela $11)$.
Encontramos relatos de uso de uma única técnica visando estenoses, independentes das etiologias, como as anoplastias com retalhos cutâneos,como anoplastia com retalho cutâneo "em S" (Ferguson ${ }^{57}$ ), anoplastia com retalho cutâneo "de Sarner" (Sarner ${ }^{66}$ ), anoplastia com retalho cutâneo "Y-V" (Ramanujan et al. ${ }^{63}$ ) e Gingold \& Arvanitis ${ }^{58}$ ), anoplastia com retalho cutâneo em "C" (Oh \& Zinberg ${ }^{60}$ ), anoplastia com avanço de retalho cutâneo "de Rosen" (Rosen ${ }^{65}$ ), anoplastia com retalho cutâneo "caseiro" (Christensen et al. ${ }^{55}$ e Sentovich et al. ${ }^{67}$ ), anoplastia com retalho cutâneo em "diamante" (Caplin \& Kodner ${ }^{54}$ ), anoplastia com retalho cutâneo "insular em U" (Pearl et al. ${ }^{61}$ e Pidala et al. ${ }^{62}$ ); as anoplastias com retalhos mucosos (Khubchandani ${ }^{59}$ ); e as anoplastias com retalhos cutâneo-mucosos (Cruz et al. ${ }^{56}$, Reis Neto et al. ${ }^{64}$, Tagliolatto ${ }^{68}$ e Wee \& Joseph $\left.{ }^{69}\right)$; todos eles admitindo bons resultados com as técnicas de preferência de cada um. Relatam tempo de cicatrização em um período de 4 a 6 semanas, à exceção de (Reis Neto et al. ${ }^{64}$ ), que observou um tempo menor (média de 18 dias). Recidivas são assinaladas em números muito baixos, relatando Reis Neto et al. ${ }^{64}$ uma única recidiva $(0,8 \%)$ com o retalho trapezóide cutâneo-mucoso em 115 pacientes portadores de estenose anal.

Incidência e abordagem da hemorragia pós-hemorroidectomia em 21 pacientes operados: deixados de lado casos de hemorragias anais póshemorroidectomia debelados com aplicação de gelo e repouso, todos os 21 pacientes que desenvolveram hemorragia anal pós-hemorroidectomia resistente a tratamento clínico $(0,9 \%)$ foram submetidos à revisão cirúrgica com ligadura e transfixação de pedículo (tabela 12). Achamos que no paciente levado à cirurgia por hemorragia anal pós-hemorroidectomia, encontrandose um pedículo apresentando sangramento importante o mesmo deve ser re-transfixado; e não sendo identificado o pedículo que sangra, todos devem ser retransfixados. Não houve como aferir este dado com a literatura.

Incidência e abordagem do agravamento da incontinência fecal pós-hemorroidectomia em 5 pacientes operados: pacientes, sobretudo idosos e multíparas que apresentam grandes processos hemorroidários, apresentam certo grau de incontinência para gases e para secreção fecal (sobretudo após defecação) e mesmo para fezes, padecendo de sensação imperiosa de defecar, cujo não atendimento pode 
resultar em passagens involuntárias de fezes pelo ânus. Sendo feito o diagnóstico de doença hemorroidária, os pacientes devem ser informados de que o quadro pode se agravar com a hemorroidectomia, de imediato, mesmo a cirurgia não interferindo com a musculatura esfincteriana. E que tal decorre do fato do canal anal estar parcialmente preenchido pelos mamilos hemorroidários, cuja remoção pode levar a um alargamento proporcional do mesmo (falta dos mamilos hemorroidários), gerando o agravamento da incontinência. Mas que, com o correr dos dias, voltando a contração esfincteriana ao normal, o quadro de incontinência deve aliviar. E se tal não ocorrer, poderia ser feita uma cirurgia corretiva do tipo esfincteroplastia em jaquetão ou em pregueamento. É muito importante que estas coisas sejam conversadas no pré-operatório, evitando dissabores no pós-operatório. Dos 5 pacientes que desenvolveram agravamento da incontinência fecal póshemorroidectomia $(0,2 \%), 2$ foram submetidos à esfincteroplastia $(40,0 \%)$ e 3 tiveram a incontinência melhorada com o tempo, sem necessidade de cirurgia $(60,0 \%)$ (tabela 13). Não houve como aferir este dado com a literatura.

Incidência e abordagem da sepse anal póshemorroidectomia em 3 pacientes operados: todos os 3 pacientes que desenvolveram sepse anal póshemorroidectomia foram levados à cirurgia para drenagem e desbridamento, com sucesso, da mesma forma que abscessos inespecíficos de outras causas (tabela 14). Não houve como aferir este dado com a literatura.

\section{CONCLUSÕES}

1. Hemorroidectomia é uma cirurgia segura (3,1\% de complicações, sem óbitos).

2. Estenose anal foi a complicação mais comum ( $p<0,05)$, na quarta década ( $>>0,05)$, em mulheres $(\mathrm{p}<0,05)$, sem hipertonia esfincteriana $(\mathrm{p}<0,05)$, com fissura ou área de fibrose $(\mathrm{p}<0,05)$, de forma anular $(\mathrm{p}<0,05)$, com aparecimento entre 11 e 30 dias de pósoperatório $(\mathrm{p}<0,05)$, mais comum por técnica aberta $(\mathrm{p}>0,05)$, sendo a cirurgia corretiva mais usada a anotomia simples com excisão de fibrose e a anoplastia com retalho cutâneo ( $p>0,05)$.

3. A hemorragia grave foi a segunda complicação ( $p>0,05)$, mais comum entre os homens ( $p<0,05)$, na quarta década $(p>0,05)$, por técnica aberta $(p>0,05)$, todos os casos submetidos à ligadura e transfixação de pedículo.

4. O agravamento da incontinência fecal foi a terceira complicação mais comum ( $p>0,05)$, na oitava década ( $p>0,05)$, por técnica fechada $(p>0,05)$, com somente 2 pacientes $(40,0 \%)$ submetidos à esfincteroplastia $(p>0,05)$.

5. Os 3 pacientes com sepse anal grave foram submetidos à drenagem e desbridamento ( $>0,05)$.

6. Houve maior incidência de complicações na $4^{\mathrm{a}}$ e na $3^{\mathrm{a}}$ décadas $(\mathrm{p}>0,05)$, ocorrendo mais entre homens $(p<0,05)$, tendo as mulheres desenvolvido mais estenose $(p<0,05)$ e agravamento da incontinência $(p>0,05)$, e os homens mais hemorragia $(p<0,05)$ e sepse anal $(\mathrm{p}>0,05)$.

ABSTRACT: The author had the opportunity in a 38-year period of practice in Coloproctology, to attend 34,000 patients. Diagnose of hemorrhoid as the main cause of the symptoms presented was achieved in 9,289 patients (27.3\%). 2,417 of the 9,289 patients $(\mathbf{2 6 . 0 \%})$ underwent hemorroidectomy. The aim of this report is to analyse these 76 patients as far as epidemiologic aspects are concerned (age and gender), surgical techniques for hemorrhoidectomy as well as surgical approach for each kind of complications. Results are correlated to the literature. Most of the patients were submitted to open hemorrhoidectomy $(2,014$ patients, $83.3 \%$ ), and only 232 patients $(\mathbf{9 . 6 \%})$ to close and 171 patients $(7.1 \%)$. There were 76 cases of surgical complications (3.1\%) being anal stricture (44 cases, $1.8 \%$ ) and postoperative bleeding ( 21 cases, $0,9 \%)$ the two most common complications, followed by worsenning of the fecal incontinence and anal sepsis ( 5 cases each, $0.2 \%$ ) besides 3 cases of systemic complications $(0.1 \%)$ and no mortality. Anal stricture was more common in four decade patients (18 cases, 2.6\%), being far more common associated to sphyncteric hipertony ( 21 cases, $70.0 \%$ ), anular in shape (31 cases, $70.4 \%$ ) developed between 11 and 30 postoperative day $(27$ cases, $61.5 \%)$ being 14 patients $(31.8 \%)$ submitted to single anotomy and fissurectomy or fibrosectomy and 7 patients being approached with anoplasty with "V" skingraft. Posoperative anal hemorrage was more common in fourth decade patients (6 cases, $1.2 \%$ ) and all of them underwent transfixation of the pedicle. Two out of 5 patients $(40.0 \%)$ with worsenning of fecal incontinence were submitted to sphynteroplasty; all patients with anal sepsis ( 3 patients) underwent drainage and debridation; and three patients with systemic complications were approached clinically.

Key words: Hemorrhoidectomia; Hemorrhoidectomia Complications; Anal Surgery. 


\section{REFERÊNCIAS}

1. Barone B, Matos D, Deak e \& Saad SS. Hemorroidectomia com o uso de diatermia e sem ligadura do pedículo. Atualização em Coloproctologia 1995: 203-205.

2. Barron (1962). In: Rebel K \& Rebel VF. Tratamento Não Cirúrgico da Doença Hemorroidária. In: Cruz GMG. Livro Texto "Coloproctologia", Volume III - "Coloproctologia Terapêutica", Editora Revinter, Rio de Janeiro, 2000; XIX; 165: $2141-2147$.

3. Bat L, Melzer E, Koler M, Dreznick Z, Shemesh E. Complications of Rubber Band Ligation of Symptomatic Internal Hemorrhoids. Dis Colon Rectum, 1993; 36: 287-290.

4. Blaisdell (1954). In: Rebel K \& Rebel VF. Tratamento Não Cirúrgico da Doença Hemorroidária. In: Cruz GMG. Livro Texto "Coloproctologia", Volume III - "Coloproctologia Terapêutica”, Editora Revinter, Rio de Janeiro, 2000; XIX; 163: 2141 - 2147.

5. Borba MR, Sobrado Jr CW \& Sokol S. Hemorroidectomia pela técnica fechada (Técnica de Sokol) - análise de 322 doentes. Rev Bras Colo-Proct 1997; 17(2): 98-100.

6. Copé R. - Etiopathogenie de la maladie hémorroïdaire Acta Gastroent.Belgica 1994 March-April; vol LVII: 206-12.

7. Corman ML. Colon \& Rectal Surgery ( $4^{\text {th }}$ edition). LippincortRaven Publishers, 1998.

8. Costa e Silva IT, Chamhic JE, Novo NF, Juliano Y, Goldenberg S. Frequiência da dor após a ligadura elástica de hemorróidas: estudo prospectivo e randomizado. Rev. Bras. Colo-Proctol., 1990; 10: 134-138.

9. Cruz GMG. Livro Texto "Coloproctologia", Volume I "Coloproctologia - Propedêutica Geral”, Editora Revinter, Rio de Janeiro, RJ, 1998.

10. Cruz GMG. Livro Texto "Coloproctologia”, Volume II "Coloproctologia - Propedêutica Nosológica", Editora Revinter, Rio de Janeiro, RJ, 1999.

11. Cruz GMG. Livro Texto "Coloproctologia", Volume III "Coloproctologia - Terapêutica", Editora Revinter, Rio de Janeiro, RJ, 2000.

12. Detrano SJ. The Role of cryosurgery in management of anorectal disease: 350 cases. Dis Colon Rectum, 1975; 18: 284-288.

13. Ferguson JA \& Heaton JR. Closed hemorrhoidectomy. Dis Colon Rectum 1959; 2: 176-9.

14. Ganchrow MI, Mazier WP, Friend WG et a.1. Hemorrhoidectomy revisited - a computer analysis of 2.038 cases. Dis Colon Rectum 1971; 14: 128-133.

15. Goligher J. Surgery of the Anus, Rectum and colon. $5^{\text {th }}$ edition. London: Bailieri -Tindall, 1984.

16. Haas P. A. and cols. - The pathogenesis of Hemorrhoids. Diseases of the Colon and Rectum, 1984 July; vol 27 (7): 442-50.

17. Haas P. A. and cols. - The prevalence of Hemorrhoids. Diseases of the Colon and Rectum, 1983 July; vol 26 (7): 435-39.
18. Hodgson WJB \& Morgan J. Ambulatory hemorrhoidectomy with CO2 laser. Dis Colon Rectum 1995; 38: 1265-1269.

19. Iwagaki H, Higuchi Y, Fuchimoto S \& Orita K. The laser treatment of hemorrhoids: results of a study on 1816 patients. Jpn J Surg 1989; 19: 658-61.

20. Jensen DM, Jutabha R, Machicado GA, Jensen ME, Cheng S, Gornbein J, Hirabayashi K, Ohning G, Randall G. Prospective randomized comparative study of bipolar eletrocoagulation versus heater probe for treatment of chronically bleeding, internal hemorrhoids. Gastrointest Endosc, 1997; 46: 435443.

21. Johanson JF, Rimm A. Optimal nonsurgical treatment of hemorrhoids: a comparative analysis of Infrared coagulation, Rubber Band ligation and Injection sclerotherapy. Am J Gastroenterol, 1992; 87: 1600-1606.

22. Lacerda Filho A \& Melo JRC. Hemorroidectomia em regime ambulatorial sob anestesia local - estudo prospectivo de 50 casos. Rev Bras Colo-Proct 1995; 15(4): 206-210.

23. Leff EI, Hemorrhoidectomy - Laser vs nonlaser: outpatient surgical experience. Dis Colon Rectum 1992; 35: 743-746.

24. Loder P. B. et al. - Haemorrhoids: pathology, pathophisiology and aetiology. Br. J. surgery 19941994 July; 81 (7): 946-54.

25. MacRae HM, McLeod JH. Comparison of hemorrhoidal treatment modalities. A Meta-Analysis. Dis Colon Rectum, 1995; 38: 687-694.

26. Mazier WP, Levien DH, Luchtefeld MA, Senagore AJ. Surgery of the Colon, Rectum and Anus, W.B. Saunders Company Ltd, 1995.

27. McConnell JC \& Khubchandani IT. Long term follow-up of closed hemorrhoidectomy. Dis Colon Rectum 1983; 226:797.

28. McLeod JH. In defense of cryotherapy for hemorrhoids: A modified method. Dis Colon Rectum, 1982; 25: 332-335.

29. Milligan ET, Morgan CN \& Jones LE. Surgical anatomy of the anal canal and the operative treatment of haemorrhoids. Lancet 1937; 2: 1119-24.

30. Mitchell. In: Rebel K \& Rebel VF. Tratamento Não Cirúrgico da Doença Hemorroidária. In: Cruz GMG. Livro Texto "Coloproctologia", Volume III - "Coloproctologia - Terapêutica”, Editora Revinter, Rio de Janeiro, 2000; XIX; 163: 2141 $-2147$.

31. Nahas SC, Sobrado CW, Marques CFS, Habr-Gama A Projeto doenças orificiais. Experiência do HCFMUSP em Hospital- dia e doenças anorretais. Em publicação na Rev Hosp Clin Fac Med S Paulo.

32. Nahas SC, Sobrado CW, Araújo SEA, Imperiale AR, HabrGama A \& Pinotti HW. Resultados do tratamento cirúrgico da doença hemorroidária em 475 doentes. Rev Hosp Clin Fac Med S Paulo 1997; 52(4): 175-179.

33. Nath, Kreitmaier e Kiefhaber (1975). In: Rebel K \& Rebel VF. Tratamento Não Cirúrgico da Doença Hemorroidária. In: Cruz GMG. Livro Texto "Coloproctologia", Volume III "Coloproctologia - Terapêutica", Editora Revinter, Rio de Janeiro, 2000; XIX; 163: 2141 - 2147. 
34. Obando RN. Hemorrhoids. An Alap 1966; 1: 110.

35. Oueidat DM, Jurjus AR. Management of hemorrhoids by Rubber Band Ligation. J Med Liban, 1994; 42: 11-14.

36. Parks AG. The surgical treatment of haemorrhoids. Br J Surg 1956; 43: 337-351.

37. Perez-Miranda M, Gomez-Cedenilla A, Leon-Colombo T, Pajares J, Mate-Jimenez J. Effect of fiber supplements on internal bleeding hemorrhoids. Hepatogastroenterology, 1996; 43: 1504-1507.

38. Ramos JR, Pinho M, Valory E, Nascimento ML \& Souza PCT. Cirurgia ambulatorial em colo-proctologia. Rev Bras coloProct 1988; 8(1): 11-13.

39. Randall GM, Jensen DM, Machicado GA, Hirabayashi K, Jensen ME, Yon S, Pelayo E. Prospective randomized comparative study of bipolar versus direct current electrocoagulation for treatment of bleeding internal hemorrhoids. Gastrointest Endosc, 1994; 40: 403-410.

40. Rebel K \& Rebel VF. Tratamento Não Cirúrgico da Doença Hemorroidária. In: Cruz GMG. Livro Texto "Coloproctologia", Volume III - "Coloproctologia - Terapêutica", Editora Revinter, Rio de Janeiro, 2000; XIX; 163: 2141 - 2147.

41. Reis Netto JA, Quilici FA, Cordeiro F, Reis Junior JA. Tratamento ambulatorial de hemorróidas: estudo prospectivo randomizado. Rev. Bras. Colo-Proctol, 1989; 9: 131-137.

42. Reis Netto JA, Quilici FA, Cordeiro F \& Reis Jr JA. Open versus semi-open hemorrhoidectomy: a random trial. Int Surg 1992; 77(2): 84-90.

43. Ruiz Moreno S. Hemorroidectomia in: $21^{\circ}$ Congresso Brasileiro de Coloproctologia 1971.33.

44. Santos HA. Doença Hemorroidária. In: Cruz GMG. Livro Texto "Coloproctologia", Volume II - "Coloproctologia Propedêutica Nosológica”, Editora Revinter, Rio de Janeiro, RJ, 1999; 75:1139 - 1156.

45. Santos RP. Etiopatogenia das hemorróidas. Rev Bras ColoProct 1993; 13(2):55-66.

46. Senagore A, Mazier WP, Lutchefeld MA, MacKeigan JM \& Wengert T. Treatment of advanced hemorrhoidal disease: a prospective, randomized comparison of cold scalpel $v s$ contact Nd: YAG laser. Dis Colon Rectum 1993; 36: 1041-9.

47. Smith LE. Hemorrhoidectomy with lasers and other contemporary modalities. Surg Clin North Am 1992; 72(3): 665-679.

48. Sobrado Jr. CW, Bringel RWA, Nahas SN \& Habr-Gama A. Cirurgia proctológica ambulatorial sob anestesia local - análise de 351 cirurgias. Rev Hosp Clin Fac Med S Paulo 1998 Volume 6 (novembro/dezembro).

49. Sobrado Jr. CW, Bringel RWA, Nahas SN. Tratamento Cirúrgico da Doença Hemorroidária. In: Cruz GMG. Livro Texto "Coloproctologia", Volume III - "Coloproctologia - Terapêutica”, Editora Revinter, Rio de Janeiro, 2000; XIX; 164: 2148 -2160 .

50. Torres P. Ligadura elástica das hemorróidas (atualização clínica). Rev. Bras. Colo-Proctol; 1983: 3: 66-68.
51. Wang JY, Chang-Chien CR, Chen JS et al. The role of laser in hemorrhoidectomy. Dis Colon Rectum 1991; 34: 78.

52. Whitehead W. The surgical treatment of haemorrhoids. Br Med J 1882; 1: 148-50.

53. Wrobleski DE. Rubberband ligation of hemorrhoids. RJ Med; 1995: 172-173.

54. Caplin DA, Kodner IJ. Repair of anal stricture and mucosal ectropion by simple flap procedures. Dis Colon Rectum, 1986; 29:92-4.

55. Christensen MA, Pitsch RM Jr, Cali RL, Blatchford GJ, Thorson AG. "House" advancement pedicle flap for anal stenosis. Dis Colon Rectum, 1992; 35:201-203.

56. Cruz GMG, Ferreira RMRS, Neves PM. Estenose Anal PósHemorroidectomia com Indicação Cirúrgica - Experiência com 75 casos. Rev Bras Coloproct, 2004; 24(1): 20 - 32.

57. Ferguson JA. Repair of "Whitehead deformity" of the anus. Surg Gynecol Obstet, 1959;108:115-116.

58. Gingold BS, Arvanitis M. Y-V anoplasty for treatment of anal stricture. Surg Gynecol Obstet, 1986;162:241-242.

59. Khubchandani IT. Mucosal advancement anoplasty. Dis colon Rectum, 1985;28:194-196.

60. Oh C, Zinberg J. Anoplasty for anal stricture. Dis Colon Rectum, 1982;25:809-810.

61. Pearl RK, Hooks VH, Abcarian H, Orsay CP, Nelson RL. Island flap anoplasty for the treatment of anal stricture and mucosal ectropion. Dis Colon rectum, 1990;33:581-583.

62. Pidala MJ, Slezak FA, Porter JA. Island flap anoplasty for anal canal stenosis and mucosal ectropion. Am Surg, 1994;60:194-196.

63. Ramanujan PS, Venkatesh KS, Cohen M. Y-V anoplasty for severe anal stenosis. Continence Surg, 1988;33:62-68.

64. Reis Neto JA, Quilici FA, Reis Junior JA. Classification and treatment of postoperative anal stenosis. Rev Bras Colo-Proct, 1987;7:7-12.

65. Rosen L. Anoplasty. Surg Clin North Am, 1988;68:1441-1446.

66. Sarner JB. Plastic relief of anal stenosis. Dis Colon Rectum, 1969;12:277.

67. Sentovich SM, Falk PM, Christensen MA, Thorson AG, Blatchford GJ, Pitsch RM. Operative results of house advancement anoplasty. Br J Surg, 1996;83:1242-1244.

68. Tagliolatto Jr L. Estenose anal cicatricial: anoplastia por deslizamento de duplo retalho. Rev Bras Colo-Proct, 1989;9:146-150.

69. Wee JT, Joseph VT. A new technique of vaginal reconstruction using neurovascular pudendal-thigh flaps. A preliminary report. Plast Reconstr Surg 1989;83:701-709.

\section{Endereço para correspondência:} GERALDO MAGELA GOMES DA CRUZ

Rua Rio de Janeiro, 2017 / 1401 - Lourdes

Belo Horizonte - MG

30160-042 